\title{
Should elliptical galaxies be idealised as collisionless equilibria?
}

\author{
Henry E. Kandrup (kandrup@astro.ufl.edu) \\ Department of Astronomy, Department of Physics, and Institute for Fundamental \\ Theory, University of Florida, Gainesville, Florida, USA 32611
}

October 26, 2018

\begin{abstract}
This review summarises several different lines of argument suggesting that one should not expect cuspy nonaxisymmetric galaxies to exist as robust, longlived collisionless equilibria, i.e., that such objects should not be idealised as timeindependent solutions to the collisionless Boltzmann equation.
\end{abstract}

Keywords: galaxies: kinematics and dynamics

\section{Motivation}

Should elliptical galaxies be visualised as objects that spend most of their lives in or near a true equilibrium, continually disturbed somewhat by nearby objects, but not exhibiting any systematic dynamical evolution? Or should they be viewed instead as objects that may be close to some equilibrium, but are drifting through phase space in such a fashion as to manifest a systematic secular evolution on time scales shorter than $t_{H}$, the age of the Universe? In other words: once they have settled down towards a near-equilibrium, should galaxies be viewed as static or dynamic entities?

Over the age of the Universe, ellipticals clearly change in terms of such properties as colour. However, it is probably fair to say that, until recently, many, if not most, astronomers have typically ignored the possibility of systematic dynamical changes except, perhaps, in response to very close galaxy-galaxy encounters, arguing, e.g., that discreteness effects (i.e., gravitational Rutherford scattering between individual stars) should be unimportant since the natural relaxation time $t_{R} \gg t_{H}$. The object of this review is to argue that this conventional wisdom may not be completely correct. Complex equilibria, especially cuspy and/or nonaxisymmetric equilibria, may not exist or may be very hard for a real galaxy to find; and, especially in high density environments, external irregularities may be significantly more important in triggering systematic changes in the bulk structure of a galaxy than has been generally recognised hitherto.

(C) 2018 Kluwer Academic Publishers. Printed in the Netherlands. 


\section{Can realistic equilibrium models exist?}

Dating back to the pioneering work of Eddington and Jeans, galactic dynamicists have typically tried to construct equilibrium solutions to the collisionless Boltzmann equation $(C B E)$ using global integrals like the energy $E$ and rotational angular momentum $J_{z}$. Since these quantities are conserved, any function of them will also be conserved, so that they can be used to define an equilibrium phase space density, or distribution function, $f_{0}$. However, such 'standard' equilibria are problematic if one allows for the possibility that many galaxies are at least moderately triaxial, as one seems compelled to do from an analysis of observations ( $c f$. Tremblay and Merritt 1995) Generic time-independent, albeit nonaxisymmetric, potentials admit only one continuous symmetry, namely time-translation, so that there is only one global integral. If the system is nonrotating, this corresponds to the energy $E$; if, instead, the system is in uniform rotation, this corresponds to the Jacobi integral $E_{J}$.

The problem, then, is that it seems impossible to model triaxial configurations with a strong central condensation in terms of equilibrium solutions $f_{0}(E)$ or $f_{0}\left(E_{J}\right)$. One knows, e.g., that every $f_{0}(E)$ must correspond to a spherically symmetric configuration (Perez and Aly 1996). A rotating solution $f_{0}\left(E_{J}\right)$ can in principle correspond to a triaxial configuration, but such configurations seem unrealistic ( $c f$. Vandervoort 1980, Ipser and Managan 1981). Physically these triaxial configurations can exist for the same reason as the triaxial Riemann ellipsoids, namely because it is energetically favourable for the configuration to deviate from axisymmetry. However, this requires rotation rates that are too large to be realistic and central condensations much smaller than those observed in real early-type galaxies (cf. Lauer et al 1995). For example, triaxial equilibria with distribution functions appropriate for a rotating polytrope cannot have a central density larger than about 3.12 times the mean density.

One can of course seek to construct models with 'unobvious' symmetries such as, e.g., models corresponding to integrable Stäckel potentials. However, nonaxisymmetric models admitting two or three global integrals are extremely nongeneric, possessing continuous symmetries whose physical origins are far from obvious. Three-integral potentials constitute a set of measure zero in the set of genuinely threedimensional potentials and, as such, if one wants to model real galaxies with such integrable potentials, it would seem crucial to identify a guiding principle which would explain why it is that nature selects such equilibria. One might perhaps hope to model triaxial galaxies in terms of potentials that admit only two global integrals. However, it is not 
clear whether there exist realistic potentials that admit only two global integrals which are not axisymmetric; and, even presuming that such potentials exist, these would again seem comparatively nongeneric.

Alternatively, one can look for equilibria involving 'local integrals' (cf. Lichtenberg and Lieberman 1992), the conserved quantities that make regular orbits behave as if they were integrable, even if the potential is nonintegrable and chaotic orbits also exist (Kandrup 1998a). One can, e.g., try to construct equilibria which contain both regular and chaotic orbits but which, for fixed $E$ or $E_{J}$, assign different weights to the regular and chaotic phase space regions. This is, in fact, the tack implicit in almost all work with nonintegrable potentials that break axisymmetry, including the triaxial generalisations of the Dehnen (1993) potentials considered, e.g., by Merritt and Fridman (1996).

This problem can be addressed numerically using some variant of Schwarzschild's (1979) method. What this entails is (i) specifying the presumed mass density $\rho$ and gravitational potential $\Phi$ for some timeindependent equilibrium, (ii) generating a huge library of orbits evolved in this potential, and then (iii) trying to select a weighted ensemble of orbits from that library which reproduces the assumed density. Only by demanding that this weighted ensemble reproduce the original $\rho$ can one ensure that one has a true self-consistent equilibrium, in which the matter in the galaxy evolves in the potential generated by the matter itself.

The usual implementation of this method might seem problematic in that it ignores the role of conserved quantities like energy. However, it was shown some years ago (Vandervoort 1984) that these quantities are really hiding in the method, at least for the special case of integrable potentials, and more recently Kandrup (1998a) has shown that this method can also be modified in a natural fashion to incorporate 'local integrals'. The key observation is that it is not orbits per se that should be considered as the fundamental ingredients. Rather, the crucial point is to select a collection of time-independent building blocks which, being time-independent, can be used as static constituents for a timeindependent equilibrium. Very recently, Copin, Zhao, and de Zeeuw (2000) have shown how, for the special case of an integrable potential, one can actually proceed semi-analytically, provided only that actionangle variables can be implemented explicitly: There is a one-to-one correspondence between values of the actions and time-independent density building blocks; and, by sweeping through all possible values of the actions, one is guaranteed to consider all possible time-independent building blocks. However, it does not seem possible to generalise this approach to nonintegrable potentials. 
Unfortunately, there are obvious problems with Schwarzschild's method or any other numerical scheme. In particular, there is no proof that, for any given $\rho$, a self-consistent equilibrium exists or that that equilibrium is unique. Indeed, even assuming that exact solutions do exist, there is no guarantee that a numerical 'solution' is a reasonable approximation to some real solution. Whether or not an equilibrium exists, the numerical prescription will find some 'best fit' solution, and there is no reason a priori to assume that that 'best fit' corresponds to a bona fide collisionless equilibrium. Alternatively, the inability to generate anything remotely resembling a 'reasonable' solution need not guarantee that no solution exists: this may simply signal an incomplete orbital library. At the present time, the best that one might hope to do is sample one's purported equilibria to generate $N$-body realisations, evolve these $N$-body realisations into the future, and then determine whether these behave more or less stably for a finite time. However, even this is problematic. The normal discretisation involved in implementing Schwarzschild's method is so coarse that $N$-body realisations generated from Schwarzschild models of stable equilibria like Plummer spheres can behave unstably Siopis (2001).

Another, potentially even more serious, problem with almost all work hitherto is that the density distributions which have been considered are highly idealised. Most Schwarzschild modeling has involved an assumption of strict axisymmetry or strict ellipsoidal symmetry with constant axis ratios. For example, the claim that triaxial equilibria cannot exist for galaxies with very steep cusps is based almost completely on an analysis of the triaxial generalisations of the Dehnen potentials (Merritt and Fridman 1996). However, it is by now well established that real ellipticals tend to have distinctly disky or boxy isophotes, the details of which correlate with other properties of the galaxy, such as the steepness of the central density cusp or the bulk rotation rate (cf. Kormendy and Bender 1996). Moreover, even if a galaxy is nearly axisymmetric in the center, as seems likely for the coreless ellipticals, they could be distinctly non-axisymmetric in their outer regions. The claim that triaxial potentials containing a very large supermassive black hole have 'too many' chaotic orbits ( $c f$. Merritt and Valluri 1999) may well reflect unnatural attempts to combine potentials with incompatible symmetries ( $c f$. Kandrup and Sideris 2001a): the black hole is presumably nearly spherical or axisymmetric, but one typically assumes that the surrounding galaxy is triaxial with fixed axis ratios down to very small scales.

In any event, the fact that real galaxies are disky or boxy is probably no accident, and it would seem that realistic galactic models should become more nearly axisymmetric towards the center. However, such 
'more complex' objects might seem even less likely to manifest continuous symmetries that give rise to global integrals, so that, assuming that they exist, equilibria with such shapes would be even more likely to rely on 'local integrals'.

\section{Will real galaxies evolve towards such equilibria?}

Over the past several decades, considerable effort, both analytic and numerical, has been devoted to the construction of equilibrium solutions to the collisionless Boltzmann equation. Unfortunately, however, much less is known about a time-dependent evolution governed by the $C B E$. There is, e.g., no proof that generic initial data will evolve towards a time-independent equilibrium, even assuming that the configuration is gravitationally bound. Even such a basic property as global existence, i.e., the fact that $f(t)$ does not evolve singularities and/or caustics, was only proven in the early 1990's (Pfaffelmoser 1992, Schaeffer 1991). It would seem that the only hard results about a time-dependent evolution that have been established to date concern the behaviour of quantities like time-averaged moments in an asymptotic $t \rightarrow \infty$ limit ( $c f$. Batt 1987). That so little is known is not really surprising. Even for the seemingly simple case of mechanical systems with short range forces, it is often very difficult, if not impossible, to prove that there is any approach towards equilibrium ( $c f$. Sinai 2000).

In this regard, it should be emphasised that there exist exact timedependent solutions to the $C B E$ which do not manifest any approach towards an equilibrium. For example, Louis and Gerhard (1988) used semi-analytic techniques to construct a solution which corresponds to finite amplitude, undamped oscillations about an otherwise timeindependent equilibrium $f_{0}$. Moreover, at least for the toy model of onedimensional gravity, counter-streaming initial conditions, corresponding to a collision between two galaxies initially in equilibrium, can yield a numerical evolution towards a final state with undamped oscillations (cf. Mineau, Feix, and Rouet 1990). A priori it might seem surprising that such oscillations do not exhibit linear and/or nonlinear Landau damping which would cause them to phase mix away. The crucial point physically is that these solutions contain 'phase space holes,' i.e., regions in the middle of the otherwise occupied phase space regions where $f \rightarrow 0$, so that one has the possibility of excitations that do not undergo a particle-wave interaction.

The idea that oscillations never damp may seem too extreme to be realistic. However these results would suggest that internal irregularities could persist much longer than the theorist is wont to assume. 
In any event, because an evolution governed by the $C B E$ is Hamiltonian ( $c f$. Kandrup 1998c), any statement regarding an approach towards equilibrium must entail a coarse-grained description of the system. This could, e.g., involve a consideration of coarse-grained distribution functions, as in the original proposal of violent relaxation (Lynden-Bell 1967). Alternatively, one might consider the evolution of a collection of lower order moments, e.g., in the context of a cumulant expansion, an approach well known from plasma physics and accelerator dynamics. In either setting, the obvious question is: how fast, and how completely, do the observables of interest - either coarse-grained distributions or lower order moments - approach time-independent values?

In a galaxy that is far from equilibrium, it is not unlikely that many orbits will exhibit an exponentially sensitive dependence on initial conditions, and the resulting chaotic mixing (cf. Kandrup and Mahon 1994, Merritt and Valluri 1996, Kandrup 1998b) will certainly help a galaxy shuffle itself up. However, there is no guarantee that the galaxy will settle down all that efficiently towards a time-independent or nearly time-independent equilibrium! Indeed, recent numerical simulations ( $c f$. Vesperini and Weinberg 2000) suggest that close encounters between galaxies can lead to long-lived pulsations. (See also the oscillations described in Miller and Smith 1994.) It will be argued below that such oscillations could in fact trigger secular evolution on a time scale $<t_{H}$.

Analyses of flows in a fixed potential suggest ( $c f$. Kandrup 1998b) that, when evolved into the future, generic ensembles of initial conditions do eventually exhibit a coarse-grained approach towards equilibrium. However, this can be an extremely complex, multi-stage process for nonintegrable potentials with a phase space that admits a complex coexistence of both regular and chaotic regions and, consequently, is riddled by a complex Arnold web. In particular, it is apparent that the rate of approach towards equilibrium can depend sensitively on the level of coarse-graining: probing the system at different scales and/or in terms of different order moments can lead to significant differences in the rate associated with any approach towards equilibrium. This is hardly surprising given the physical expectation that, as a result of phase mixing, power should cascade from larger to shorter scales.

The obvious point in all this is that great care must be taken in deciding what one ought to mean by asserting that a galaxy is 'nearly in equilibrium.' A galaxy could, e.g., look 'nearly in equilibrium' when viewed in terms of its bulk properties, but still exhibit significant shorter scale variability and, most importantly, still be distinctly 'out of equilibrium' from the standpoint of dynamics. 
A crucial point, then, is that systems that have not achieved a true time-independent equilibrium tend to be more susceptible to external stimuli than systems that are in a true equilibrium. This is especially true for complex systems characterised by a six-dimensional phase space that admits a complex coexistence of regular and chaotic regions, with structures like bars or cusps that rely on an intricate balance of 'sticky' ( $c f$. Contopoulos 1971) and wildly chaotic orbits.

Real galaxies, of course, are not strictly collisionless, and it is clear that dissipative gas dynamics must have played an important role in the earliest stages of galaxy formation where, even allowing for large quantities of nonbaryonic dark matter, much of the (proto-)galaxy is comprised of dissipative gas which has not yet been converted into stars. The obvious point, then, is that dissipative effects associated with this gas could play an important role in driving the system towards a true equilibrium. Indeed, such dissipative effects, which should be more important in the higher density central regions, might drive the central regions of a cuspy galaxy towards a state that is much more nearly axisymmetric than the lower density, outer portions of the galaxy.

Nevertheless, even if dissipation is a crucial element for the evolution of primordial equilibria or near-equilibria, it would seem comparatively unimportant when considering the effects of recent collisions and other close encounters between ellipticals. Over the past decade or so, it has been recognised that ellipticals are not as gas-poor as was originally believed. However, most of the gas in ellipticals exists at high temperatures and low densities, so that its dissipative effects should be minimal. It seems implausible that dissipative gas dynamics could play a dominant role in how elliptical galaxies readjust themselves after a strong encounter with another galaxy.

However, dissipative gas dynamics is not the only physical effect which is ignored by the $C B E$. Real galaxies are also subjected to a variety of other perturbations which could in principle be important. For example, as stressed, e.g., by Merritt and Fridman (1996), the central regions of cuspy galaxies are so dense that the relaxation time $t_{R}$ associated with gravitational Rutherford scattering between neighbouring stars can be less than or comparable to $t_{H}$. And similarly, galaxies are subjected continually to perturbations reflecting the effects of companion galaxies and other nearby objects which, especially in high density clusters, can be appreciable.

An obvious question, therefore, is: what are the potential effects of ongoing low amplitude perturbations? On the one hand, one might argue that they will interfere with a systematic approach towards equilibrium since they imply that the density distribution necessarily varies in time. On the other, one might argue that these perturbations actu- 
ally expedite the approach towards equilibrium, since the time-varying forces to which the galaxy is subjected could facilitate violent relaxation by accelerating phase space transport. In particular, it would not seem completely implausible to argue that, in many cases, galactic evolution should be viewed as a two-stage process. Early on a galaxy could have evolved towards a state which, albeit not a true equilibrium, would persist as a near-equilibrium for times $>t_{H}$ in the absence of any irregularities. However, such irregularities are always present, and they could act to trigger a secular evolution, e.g., driving the system more nearly towards a true equilibrium.

\section{What are the effects of ongoing perturbations?}

Suppose that, after some more or less effective period of violent relaxation, an elliptical has settled down towards (albeit not necessarily to) some realistic complex equilibrium or near-equilibrium. That configuration could well be distinctly nonaxisymmetric, at least in the outer regions, and, if so, might be expected to exhibit variable axis ratios, becoming more nearly axisymmetric near the center. However, the gravitational potential associated with such a complex configuration is likely to involve significant measures of both regular and chaotic orbits. It is in fact well known to nonlinear dynamicists that less symmetric potentials tend to exhibit much larger measures of chaos, a point first stressed in the galactic dynamics community by Udry and Pfenniger (1988). Even comparatively simple potentials like the three-dimensional logarithmic potentials, with $V=\frac{1}{2} v_{0}^{2} \log \left(1+x^{2} / a^{2}+y^{2} / b^{2}+z^{2} / c^{2}\right)$, admit chaotic orbits for certain choices of parameter values. Indeed, there exist selfconsistent axisymmetric equilibria which manifest chaotic meridional motions, including the scale-free models considered by Evans (1994) and various spheroidal models considered by Hunter et al (1998).

The important point, then, is that the phase space associated with a time-independent three-dimensional potential that admits significant measures of both regular and chaotic orbits tends to be very complex, being laced with cantori and/or an Arnold web. The existence of this complex structure implies that the approach towards equilibrium can be comparatively inefficient. In particular, orbits could well get trapped in localised phase space regions for very long times, even though their motion is not blocked by a conservation law (like conservation of energy): In principle, an orbit may be able to access a large phase space region, but it may have to leak through a narrow 'bottleneck' to get from one part of the accessible region to another, penetrating through what the nonlinear dynamicist would call an entropy barrier. 
This suggests the possibility that a system could evolve towards a state which, albeit not a true equilibrium, could persist for times $\gg t_{H}$, at least in the absence of significant perturbations. Indeed, this possibility has been invoked by a number of authors ( $c f$. Patsis, Athanassoula, and Quillen 1997) in the context of spiral galaxies, and corresponds to what Merritt and Fridman (1996) termed 'quasi-equilibria' involving chaotic building blocks that are only 'partially mixed'.

The important observation, however, is that such configurations can be surprisingly vulnerable to even very weak irregularities. This fact was apparently first recognised more than thirty years ago in the context of simple maps ( $c f$. Lieberman and Lichtenberg 1970); and, over the past several decades has proved to be very important in the context of plasma physics and accelerator dynamics ( $c f$. Tennyson 1979, Habib and Ryne 1995), where one deals with beams of charged particles that are confined by imperfect magnetic fields and in which discreteness effects, i.e., Rutherford scattering, can be surprisingly important.

But what can low amplitude perturbations actually do? Even if the perturbations are too weak to significantly impact the values of the energy or any other collisionless invariants, they can induce systematic phase space flows on the (nearly) constant energy surfaces, e.g., by allowing orbits originally trapped in localised phase space regions to become untrapped ( $c f$. Mahon et al 1995, Kandrup, Pogorelov, and Sideris 2000) Moreover, under appropriate circumstances, perturbations can prove strong enough to induce nontrivial changes in the orbital energy and other collisionless invariants ( $c f$. Kandrup 2001).

Such perturbations act via a resonant coupling between the frequencies of the perturbation and the frequencies of the orbits (Pogorelov and Kandrup 1999). For regular orbits, the power is concentrated at a few special frequencies whereas, for chaotic orbits, power is typically broader band. In either case, however, when subjected to a perturbation characterised by its own set of natural frequencies, there is the possibility of resonance; and, to the extent that this resonance is strong, the perturbation will have a substantial effect

One might suppose that the response of the orbits to various influences will depend sensitively on the precise form of the perturbation, so that believeable computations would require a knowledge of details that are difficult, if not impossible, to extract from observations. In point of fact, however, the details seem comparatively unimportant. Because the perturbations act via a resonant coupling, all that really seems to matter are the amplitude of the perturbation, which determines how hard the orbits are being 'hit,' and the natural frequencies of the perturbation, which determine the degree to which a resonance is possible. 
The crucial point about this susceptibility to low amplitude irregularities is that changes in the orbital density and/or collisionless invariants will in general yield changes in the bulk potential and, as such, could trigger systematic evolutionary effects. It could be that the system will react in such a fashion as to stabilise itself, but it might equally well start to exhibit systematic changes in its bulk properties.

The most detailed investigation of the effects of low amplitude perturbations in the context of elliptical galaxies that has been effected hitherto (Siopis and Kandrup 2000, Kandrup and Siopis 2001) involved an investigation of flows in the triaxial generalisations of the Dehnen potentials, with or without a central supermassive black hole. These potentials are not completely realistic since they assume strict ellipsoidal symmetry with fixed axis ratios, and ignore completely the possibility of rotation. However, they do at least incorporate a high density central concentration and, as might be expected physically, they include large measures of both regular and chaotic orbits. Several different physical effects were considered:

- Discreteness effects, i.e., gravitational Rutherford scattering between individual stars, were modeled as resulting in dynamical friction and Gaussian white noise, i.e., near-instantaneous impulses, in the spirit of Chandrasekhar (1943). Even if the relaxation time $t_{R}$ on which the energy of individual orbits changes is long compared with $t_{H}$ which, as stressed by Merritt and Fridman (1996), is not always so, such encounters can still be important by accelerating diffusion on the nearly constant energy hypersurface ( $c f$. Habib, Kandrup, and Mahon 1997, Kandrup, Pogorelov, and Sideris 2000).

- The effects of satellite galaxies and companion objects were modeled as near-periodic driving, characterised by a perturbation $V=V\left(\left\{\omega_{i}\right\} t\right)$, for some small number of frequencies $\left\{\omega_{i}\right\}$.

- In a dense cluster environment, a galaxy will be impacted by a large number of different neighbouring galaxies in a fashion which is likely to be far from periodic. It thus seemed reasonable to model such an environment by allowing for coloured noise, this corresponding ( $c f$ van Kampen 1981) to a series of random impulses of finite duration. (Mathematically, coloured noise can be viewed as a superposition of periodic disturbances with different frequencies combined with random phases.) - In addition to companion objects, a galaxy can be impacted by tidal forces associated with the bulk cluster potential which, depending on the form of the potential, could be nearly periodic or largely random.

- Coherent internal oscillations, associated with a small number of normal or pseudo-normal modes, were again modeled as inducing a near-periodic driving. 
- Incoherent internal oscillations, associated, e.g., with a large number of higher order modes, were again modeled as coloured noise, i.e., nearrandom perturbations of finite duration. (Making a sharp distinction between coherent and incoherent oscillations is admittedly somewhat ad hoc.)

Given the usual assumption that the noise under consideration is Gaussian, its statistical properties are characterised completely by a knowledge of its first two moments. For both white and coloured noise, it was assumed that the average force vanishes identically, so that $\left\langle F_{a}(t)\right\rangle=0$ for $a=x, y, z$. White noise, which involves instantaneous kicks, is characterised by an autocorrelation function

$$
\left\langle F_{a}\left(t_{1}\right) F_{b}\left(t_{2}\right)\right\rangle=2 D \delta_{a b} \delta_{D}\left(t_{1}-t_{2}\right) .
$$

Coloured noise requires 'fuzzing out' the Dirac delta. As a simple example, this was done by sampling an Ornstein-Uhlenbeck process, for which

$$
\left\langle F_{a}\left(t_{1}\right) F_{b}\left(t_{2}\right)\right\rangle=\left(D / t_{c}\right) \delta_{a b} \exp \left(-\left|t_{1}-t_{2}\right| / t_{c}\right) .
$$

In each of these expressions, $D$ is the diffusion constant which, in the white noise limit, enters into a Fokker-Planck description:

$$
D \equiv \int_{0}^{\infty} d \tau\left\langle F_{a}(0) F_{a}(\tau)\right\rangle
$$

The quantity $t_{c}$ is the autocorrelation time, which represents the characteristic time scale on which the random forces change significantly.

The effects of low amplitude perturbations can be decomposed, at least approximately, into (i) motions that involve little or no changes in the energy and any other quantities which would be invariant for motion in a fixed time-independent potential; and (ii) changes in the values of the energy and any other collisionless invariants.

Even if the perturbations are so weak that such collisionless invariants as $E$ or $E_{J}$ are nearly conserved, they can significantly accelerate phase space diffusion of chaotic orbits on the nearly constant energy surface. What this entails is orbits leaking through topological partial obstructions associated with cantori and/or an Arnold web in a fashion strongly reminiscent of the standard problem of effusion, whereby gas molecules can leak through a tiny hole in a wall. If one considers an ensemble of 'sticky' orbits ( $c f$. Contopoulos 1971) initially localised in a given phase space region bounded by such obstructions, their escape is well modelled as a Poisson process ( $c f$. Pogorelov and Kandrup 1999, Kandrup, Pogorelov, and Sideris 2000), for which the number of orbits that remain trapped decreases exponentially, i.e.,

$$
N(t)=N_{0} \exp (-\Lambda t)
$$


For a specified choice for the form of the perturbation, the value of $\Lambda$ scales logarithmically in amplitude, so that, e.g., for the case of noise there is a logarithmic dependence on $D$. The effects tend to be significant provided only that the characteristic time scale associated with the perturbation is not large compared with the natural time scale associated with the orbits. For the case of coloured noise, the precise choice of autocorrelation time seems immaterial for $t_{c}<t_{D}$, but the effects of the perturbation diminish significantly for $t_{c} \gg t_{D}$.

Under appropriate circumstances, the perturbations can also induce nontrivial changes in such collisionless invariants as the energy. For the case of periodic driving, where the perturbations are characterised by a few special frequencies, the detailed effects of the perturbation can be comparatively complex ( $c f$. Kandrup, Abernathy, and Bradley 1995). However, for the case of 'random' disturbances, idealised as noise, the picture is quite simple, with the perturbations acting to trigger a diffusion process. For the case of white or near-white noise with $t_{c} \ll t_{D}$, the physics is identical to Brownian motion, as proposed originally by Chandrasekhar (1943) to model the effects of gravitational Rutherford scattering between individual stars or by Spitzer and Schwarzschild (1951) to account for the scattering of disc stars off of giant molecular clouds. For $t_{c}>t_{D}$ the effects once again begin to be suppressed. For the special case of coloured noise sampling the Ornstein-Uhlenbeck process, the rms change in energy is in general well fit by a scaling relation of the form (Kandrup 2001)

$$
\frac{\delta E_{r m s}}{|E|} \sim\left(\frac{D t}{|E|}\right)^{1 / 2} \times\left\{\begin{array}{cl}
1 & \text { for } t_{c}<t_{D} \\
\left(\frac{t_{D}}{t_{c}}\right) & \text { for } t_{c}>t_{D}
\end{array}\right.
$$

Overall, regular and chaotic orbits are affected in a nearly identical fashion, except for very large values of $t_{c}$, where regular orbits prove to be somewhat more susceptible than are chaotic orbits.

The important point in all this is that realistic choices of $D$ and $t_{c}$ can actually have a significant effect. The diffusion constant scales as $D \sim F^{2} t_{c}$, where $F$ denotes the typical size of the random forces, so that its amplitude is readily estimated.

On the nearly constant energy hypersurface, discreteness effects corresponding to a relaxation time as long as $t_{R} \sim 10^{6}-10^{7} t_{D}$ or even more can have big effects within a time as short as $100 t_{D}$. Similarly, even ignoring the effects of single very close encounters, random interactions with nearby galaxies in an environment where the separation between galaxies is ten times the size of an individual galaxy, i.e., $R_{\text {sep }} \sim 10 R_{\text {gal }}$, so that the autocorrelation time $t_{c} \sim 10 t_{D}$, can have 
appreciable effects within $100 t_{D}$ (Siopis and Kandrup 2000, Kandrup and Siopis 2001).

As regards changes in the energy and other collisionless invariants (Kandrup 2001, Kandrup and Siopis 2001), incoherent internal oscillations at the $1 \%$ level, far too small to be detected observationally, can trigger $10 \%$ changes in the energy within $100 t_{D}$; and $10 \%$ oscillations can trigger $30 \%$ changes in a comparable time. Random interactions between neighbouring galaxies are less important, at least directly, since an environment with $R_{\text {sep }} \sim 6 R_{\text {gal }}$ and $t_{c} \sim 6 t_{D}$ will only trigger $10 \%$ changes within $100 t_{D}$. However, such interactions could still prove important ( $c f$. Vesperini and Weinberg 2000) by triggering random incoherent oscillations that induce more substantial changes in the invariants.

\section{Discussion}

A major issue all too often unaddressed is the extent to which, as has been assumed here, real galaxies characterised by the highly irregular potential associated with a large number of nearly point mass stars can in fact be approximated by a smooth three-dimensional potential. Should one, for example, really expect to see effects like 'stickiness' or phase space diffusion, which have been studied primarily in the context of smooth two- and three-dimensional potentials, in real many-body systems? Indeed, it is clear that the continuum approximation misses at least some physical effects. It has, for example, become evident from both numerical computations ( $c f$. Goodman, Heggie, and Hut 1993) and rigorous analytics (Pogorelov 2001) that, even for very large $N$, individual orbits in an $N$-body system typically have large positive Lyapunov exponents, even if the $N$-body system corresponds to an integrable density distribution such as a spherical equilibrium.

However, recent numerical work involving orbits and orbit ensembles evolved in fixed $N$-body realisations of continuous density distributions has shown that, in many respects, such 'frozen- $N$ ' orbits are indistinguishable from orbits evolved in the continuous density distribution in the presence of friction and (nearly) white noise (Kandrup and Sideris 2001b, Sideris and Kandrup 2001).

Even though the Lyapunov exponents for frozen- $N$ orbits do not appear to converge towards the values assumed by orbits in the potential associated with the smooth density distribution, there is a precise sense in which, as $N$ increases, frozen- $N$ trajectories remain 'close to' smooth potential characteristics with the same initial conditions for progressively longer times. Viewed macroscopically, for both regular 
and chaotic initial conditions, frozen- $N$ trajectories and smooth potential characteristics with the same initial condition typically exhibit a linear divergence: Their mean separation $\delta r(t)$ is well fit by a growth law $\delta r / R=A\left(t / t_{G}\right)$, where $R$ represents the size of the configuration space region accessible to the orbits and $A$ is a constant of order unity. For regular orbits, $t_{G} / t_{D} \propto N^{1 / 2}$; for chaotic orbits, $t_{G} / t_{D} \propto \ln N$. Moreover, for sufficiently large $N$ ensembles of frozen- $N$ orbits can exhibit 'stickiness' qualitatively similar to what has been observed for orbits in smooth potentials. And finally, both in terms of the statistical properties of orbit ensembles and in terms of the pointwise properties of individual orbits, discreteness effects associated with a frozen- $N$ system can be well mimicked by white noise with a diffusion constant exhibiting (at least approximately) the $N$-dependence predicted when these effects are modeled as a sequence of incoherent binary encounters (cf. Chandrasekhar 1943).

The extent to which the smooth potential approximation remains valid in the context of a self-consistent $N$-body evolution is much more difficult to probe directly. However, recent work in the context of charged accelerator beams, where particles interacting via a repulsive $1 / r^{2}$ force are confined by an externally imposed potential, appears encouraging. In particular, fully self-consistent grid code simulations of intensed charged beams (which admittedly suppress the chaos associated with close encounters between individual particles) have been found to exhibit both regular and chaotic phase mixing qualitatively similar to what has been observed in smooth two- and three-dimensional potentials (Kishek et al 2001). Experiments to search for these effects in real particle beams are currently in the planning stage (Bohn et al 2002) and analogous computations for self-gravitating systems are underway.

The principal message of this review is that it may be oversimplistic to assume that elliptical galaxies should be viewed as collisionless equilibria. Realistic equilibria, corresponding to nonaxisymmetric systems which contain a high density central region, break strict ellipsoidal symmetry, and manifest variable axis ratios, may not exist and, even if they do exist, may be very difficult for real galaxies to find. Moreover, even if a galaxy seems 'close to' equilibrium observationally, it could well be comparatively 'far from' equilibrium from the standpoint of dynamics. Comparatively unsymmetric galaxies, characterised by a complex phase space that admits a coexistence of significant measures of both regular and chaotic orbits, can be surprisingly susceptible to low amplitude perturbations of the form that act in the real world and, as such, might be expected to exhibit systematic evolutionary effects over time scales $<t_{D}$. What precisely these evolutionary effects could 
be is not yet completely clear. However, there is good reason to believe that ongoing observational programs, such as the Sloan Digital Sky Survey, will provide at least partial answers to this basic question.

\section{Acknowledgements}

I am pleased to acknowledge useful collaborations with Robert Abernathy, Brendan Bradley, Barbara Eckstein, Salman Habib, Ilya Pogorelov, Ioannis Sideris, Christos Siopis, and, especially, Elaine Mahon, who first stimulated me to think about the issues discussed in this paper. This research was supported in part by NSF AST-0070809 and by the Institute for Geophysics and Planetary Physics at Los Alamos National Laboratory.

\section{References}

J. Batt, Transport Theory and Statistical Physics 16: 763, 1987

C. L. Bohn, I. V. Sideris, H. E. Kandrup, and R. A. Kishek, In Proceedings of DESY 2002, in press (2002)

S. Chandrasekhar, Rev. Mod. Phys. 15: 1, 1943

G. Contopoulos, Astron. J. 76: 147, 1971

Y. Copin, H. S. Zhao, and P. T. de Zeeuw, Mon. Not. R. astr. Soc. 381: 781, 2000

W. Dehnen, Mon. Not. R. astr. Soc. 265: 250, 1993

N. W. Evans, Mon. Not. R. astr. Soc. 267: 333, 1994

J. Goodman, D. Heggie, and P. Hut, Astrophys. J. 415: 715, 1993

S. Habib and R. Ryne, Phys. Rev. Lett. 74: 70, 1995

S. Habib, H. E. Kandrup, and M. E. Mahon, Astrophys. J. 480: 155, 1997

C. Hunter, B. Terzic, A. M. Burns, D. Porchia, and C. Zink, Ann. N. Y. Acad. Sci. 867: 61, 1998

J. R. Ipser and R. A. Managan, Astrophys. J. 250: 362, 1981

H. E. Kandrup, Mon. Not. R. astr. Soc. 299: 1139, 1998a

H. E. Kandrup, Mon. Not. R. astr. Soc. 301: 960, 1998b

H. E. Kandrup, Astrophys. J. 500: 120, 1998c

H. E. Kandrup, Mon. Not. R. astr. Soc. 323: 681, 2001

H. E. Kandrup, R. A. Abernathy, and B. O. Bradley, Phys. Rev. E 51: 5287, 1995

H. E. Kandrup and M. E. Mahon, Phys. Rev. E 49: 3735, 1994

H. E. Kandrup, I. V. Pogorelov, and I. V. Sideris, Mon. Not. R. astr. Soc. 311: 719, 2000

H. E. Kandrup and I. V. Sideris, Celestial Mechanics, in press, 2001 (astro-phy/ 0111517 )

H. E. Kandrup and I. V. Sideris, Phys. Rev. E, in press, 2001 astro-phy/0103325

H. E. Kandrup and C. Siopis, in preparation, 2002

R. A. Kishek, C. L. Bohn, I. Haber, P. G. O'Shea, M. Reiser, and H. Kandrup, In 2001 IEEE Particle Accelerator Conference, Evanston, IEEE Press, in press, 2001

J. Kormendy and R. Bender, Astrophys. J. Lett. 464: 119, 1996 
T. R. Lauer, E. A. Ajhar, Y.-I. Byun, A. Dressler, S. M. Faber, C. Grillmair, J. Kormendy, D. Richstone, and S. Tremaine, Astron. J. 110: 2622, 1995

A. J. Lichtenberg and M. A. Lieberman, Regular and Chaotic Orbits, Berlin, 1992. Springer

M. A. Lieberman and A. J. Lichtenberg, Phys. Rev. A 5: 1852, 1970

P. D. Louis and O. E. Gerhard, Mon. Not. R. astr. Soc. 233: 337, 1988

M. E. Mahon, R. A. Abernathy, B. O. Bradley, and H. E. Kandrup, Mon. Not. R. astr. Soc. 275: 443, 1995

D. Merritt and T. Fridman, Astrophys. J. 460: 136, 1996

D. Merritt and M. Valluri, Astrophys. J. 471: 82, 1996

D. Merritt and M. Valluri, Astron. J. 118: 1177, 1999

R. H. Miller and B. F. Smith, Celestial Mechanics 59: 161, 1994

P. Mineau, M. R. Feix, and J. L. Rouet, Astron. Astrophys. 228, 344, 1990

P. A. Patsis, E. Athanassoula, and A. C. Quillen, Astrophys. J. 483: 731, 1997

J. Perez and J.-J. Aly, Mon. Not. R. astr. Soc. 280: 689, 1996

K. Pfaffelmoser, J. Diff. Eqns. 95: 281, 1992

I. V. Pogorelov, University of Florida Ph. D. thesis, 2001

I. V. Pogorelov and H. E. Kandrup, Phys. Rev. E 60: 1567, 1999

J. Schaeffer, Commun. Part. Diff. Eqns. 16, 1313, 1991

M. Schwarzschild, Astrophys. J. 232: 236, 1979

I. V. Sideris and H. E. Kandrup, Phys. Rev. E, submitted (astro-phy/111488)

Ya. Sinai, private comunication, 2000

C. Siopis, private communication, 2001

C. Siopis and H. E. Kandrup, Mon. Not. R. astr. Soc. 319: 43, 2000

L. Spitzer and M. Schwarzschild, Astrophys. J. 114: 385, 1951

J. J. Tennyson, In M. Month and J. Herrara, editors, Nonlinear Dynamics and the Beam-Beam Interaction, AIP Conf. Proc. 57, 1979, p. 158

B. Tremblay and D. Merritt, Astron. J. 110: 1039, 1995

S. Udry and D. Pfenniger, Astron. Astrophys. 198: 135, 1988

P. O. Vandervoort, Astrophys. J. 240: 478, 1980

P. O. Vandervoort, Astrophys. J. 287: 475, 1984

N. G. van Kampen, Stochastic Processes in Chemistry and Physics, Amsterdam, 1981, North Holland.

E. Vesperini and M. D. Weinberg, Astrophys. J. 534: 598, 2000 